\title{
The impact of canister geometry on chemical biological radiological and nuclear filter performance: A computational fluid dynamics analysis
}

Keywords: Powered Air-Purifying Respirators, Chemical Biological Radiological and Nuclear (CBRN) filter, canister geometry, Reynolds Averaged Navier-Stokes simulations

7539 words

\section{ABSATRACT}

Steady-state axisymmetric simulations using the Reynolds-Averaged Navier-Stokes equations have been carried out in order to optimise the performance of a Chemical, Biological, Radiological and Nuclear (CBRN) canister filter for its use in a powered air-purifying respirator (PAPR). Alterations have been made to the shape of the canister, the spacing of the rear wall of the canister with regard to the carbon filter, and the bracketing between (i) the particulate filter and the carbon bed and (ii) the carbon bed and the canister wall. The pressure drop across the canister and the residence time distribution at the rear of the carbon bed have been analysed in detail based on an extensive parametric analysis involving the aforementioned variations. It has been demonstrated that the non-uniform porosity profile of the carbon bed resulted in alternating regions of high and low velocity close to the canister wall, providing a possible route for breakthrough. Designs, which included a bracket at the rear of the carbon bed, blocked this route and consequently had a longer minimum mean residence time than those, which did not. It has also been shown that the spacing between the carbon bed and the canister rear wall had a large impact on both residence time and pressure drop. In cases where the carbon backed directly onto the canister rear wall flow in the axial direction from the outside wall towards the canister axis resulted in far greater pressure drop and a reduction in minimum mean residence time within the carbon bed. 


\section{INTRODUCTION}

Air Purifying Respirators (APR) are used in hazardous environments to provide the user with a supply of clean, breathable air. This is achieved by forcing air through a series of filters that will remove any contaminants. In a conventional APR the driving force to draw air through the filter is the wearer's own inhalation, which requires a tight face seal and limits the possible resistance provided by the filter before the user becomes unable to breathe at an acceptable rate. In a Powered Air Purifying Respirator (PAPR) the air is forced through the filters by means of a blower which is powered by a battery. PAPRs maintain a positive pressure in the facepiece to prevent contaminated air from entering in the case of a leak. Air is released from the mask by means of a non-return valve when a set pressure in the mask is reached.

PAPRs can be categorised into three main types; continuous flow, multi-flow and breathresponsive. A continuous flow PAPR provides air at a fixed flowrate, which cannot be varied. This means that it may not be able to match the user's breathing requirement as it increases under intense exercise. Additionally, continuous flow is wasted when the user exhales and is detrimental to battery and filter life. Multi-flow PAPRs function in a similar way to continuous flow PAPRs except they allow the flowrate to be controlled to allow for changes in the users breathing rate. Breath-responsive PAPRs use feedback from the facepiece to detect whether or not the user is inhaling and at what rate in order to provide airflow to match the user's requirement. Providing air only as it is needed is obviously a more efficient use of battery and filter life. ${ }^{(1)}$

In a typical PAPR and the pressure rise requirement across the blower is determined by the pressure drop within the filter. Thus, the performances of the filter in terms of both pressure drop and removal of undesirable agents play key roles in the design of PAPRs. The filters on a PAPR are typically disposable canisters at the PAPR inlet. The type of canister depends on the application, with different canisters consisting of different filter materials and sizes. For CBRN 
(chemical, biological, radiological and nuclear) applications, the filter must protect adequately against a large variety of agents. Due to the highly toxic nature of some of these agents it is essential that the filter provides an extremely high level of protection and any significant breakthrough is unacceptable. ${ }^{(2)}$

Computational Fluid Dynamics (CFD) has been used in the past as a method of predicting the performance of CBRN protective equipment. ${ }^{(3)}$ It has also been previously used to optimise filters for a number of industrial applications, and has shown good results when used to predict performance in pleated filters similar to those used at the inlet of a PAPR canister ${ }^{(4,5)}$. Threedimensional (3D) CFD simulations have been used to analyse the flow features in CBRN filter canisters in a past study ${ }^{(6)}$, in which a large range of flow rates were considered. The results of these simulations were also compared to experimental measurements of pressure drop throughout the canister and a good agreement between experimental and computational results was reported. Additionally, the bracketing used to contain the carbon bed was modified in a number of small ways (closing and opening holes in the bed) to explore the impact this would have on both the residence time of air in the bed and on the pressure drop throughout the canister. It was found that small optimisations on the bracket hole distribution can have significant impact on the performance of the canister. However, the variations to the canister geometry made in this work were limited only to the hole placement in the bracket and stopped short of more substantial changes to the canister geometry. The work was also carried out based on an assumption of uniform permeability throughout the carbon bed which does not necessarily give an accurate picture of the internal structure of the canister. ${ }^{(6)}$

Axisymmetric simulations were carried out based on another design of CBRN canister with the intent of developing a model to describe adsorption throughout the canister. ${ }^{(7)}$ In this work a radial porosity profile was implemented to better describe the packing distribution of carbon throughout the carbon bed, which is described in the "Mathematical Background" section. 
The purpose of the current analysis is to consider different axisymmetric geometries of CBRN filter canisters to analyse the effects of geometrical variations on the performance of the filter in terms of pressure drop across it and the residence time distribution at the rear of the carbon bed within the filter. This is achieved by carrying out steady-state Reynolds Averaged Navier Stokes (RANS) simulations for a flow rate which is often realised in continuous flow PAPRs, and for breath-responsive PAPRs under the normal breathing pattern of a healthy individual $^{(8)}$. For CBRN filters to be used with transient flows, continuous flow tests are commonly used to provide a good, if not comprehensive, indication of canister performance, and are used for canister performance testing in NIOSH specifications. ${ }^{(9)}$ The flowrate of $50 \mathrm{~L}$ $\min ^{-1}$ was chosen based on a past study ${ }^{(10)}$ which indicates that this is the most representative continuous flowrate for canister testing. The typical axisymmetric filter geometry considered here is shown in Fig. 1.

The mathematical background and numerical implementation pertaining to this analysis will be presented in the next section. Following this, results will be presented along with the discussion. The main findings are summarised in the final section of this paper.

\section{MATHEMATICAL BACKGROUND}

For the current analysis, steady-state Reynolds-averaged Navier-Stokes (RANS) simulations have been carried out by solving Reynolds-averaged Navier-Stokes equations. ${ }^{(11)}$ Interested readers are referred to Appendix 1 for the Reynolds averaged Navier-Stokes equations and the associated turbulence model.

\section{Configurations}

A number of different variations has been made to the baseline CBRN canister design ${ }^{(7)}$ shown in Fig. 2, and these variations are schematically shown in Fig. 3. This geometry was selected as it represented a typical CBRN canister without any novel features, and there is an 
existing body of work available on adsorption throughout the canister which could be applicable to future work using variations of this geometry. ${ }^{(7)}$ A schematic representation of the simulation domain is shown in Fig. 2. This domain represents a filter such as that shown in Fig. 1 rotated by 90 degrees and considering only one half with respect to the axis of symmetry for the sake of computational economy. This design features three separate inlets leading into a chamber containing two separate layers of filter with a small gap between them, as shown in Fig. 3. These simulations allowed for fluid flow across the entire domain without representing the internal bracketing that holds the filters in place. The following changes are made to the baseline geometry (see Fig. 3(a)):

- Brackets between the carbon and particulate filter: Two different sizes of bracketing are used, with 3mm spacing (see Fig. 3b) and 6mm spacing (see Fig. 3c).

- Extending the rear of the canister past the back of the carbon filter, leaving a space between the carbon and the narrowing of the outlet. Two different lengths of extension are used: $2 \mathrm{~mm}$ (see Fig. $3 \mathrm{~d}$ ) and $6 \mathrm{~mm}$.

- Curving the outer wall of the canister towards the outlet (see Fig. 3e) with a $10 \mathrm{~mm}$ radius of curvature.

- Curving the inner wall of the canister towards the outlet (see Fig. 3f) with a $10 \mathrm{~mm}$ radius of curvature.

- Brackets to the rear of the carbon filter (at the outlet), in the cases where the rear of the canister has been extended by $6 \mathrm{~mm}$. Two different sizes of bracketing are used, with 3 mm spacing (see Fig. 3(g)) and 6 mm spacing (see Fig. 3h).

All possible variations of these changes to the geometry have been simulated, leading to a total of 60 cases.

\section{Mesh Independence}


For the aforementioned 60 variations, the mesh independence of the results has been ensured using both coarse (Mesh A) and refined (Mesh B) Cartesian meshes with non-uniform grid spacing. The grid spacing remains small close to the wall in order to resolve the turbulent boundary layer and to ensure that the maximum $y^{+}=\rho u_{\tau} \Delta / \mu$ remains smaller than 5.0 but the grid spacing gradually increases away from the wall. The number of grid points, grid spacing and grid expansion ratio for the meshes used for this analysis are summarised in Table A1 in Appendix 2. For five cases (each separate type of spacing or bracketing at the rear of the canister) an additionally refined mesh (Mesh C) was considered in order to provide an additional level of verification of mesh independence. For each geometry, mesh independence was ensured by checking that both the coarse and refined cases gave equivalent values of pressure drop from the inlet to the outlet, and mean air age at the rear of the carbon filter, to within $1 \%$. For the other geometries the number of cells in each mesh did not vary substantially from that used in the base case.

\section{Filter Equations}

The filter material in a respirator filter canister is usually modelled as a porous medium. ${ }^{(7)}$ The canister typically contains two layers of filtration media; a pleated particulate filter near the inlet to stop particulates, followed by a layer of carbon, which acts as an adsorbent. The carbon bed is held in place by bracketing or a mesh above and below. A 2D schematic of a typical filter design is shown in Fig. 1.

The pressure drop across the porous media of the two filter layers is dependent on the local permeability $K$ for a fluid of viscosity $\mu$ as described by Darcy's Law:

$\left(\frac{-\Delta \bar{p}}{L}\right)_{i}=\frac{\mu}{K} \bar{u}_{i}$

The permeability of the particulate filter may vary over time as the particulate loading increases, although in this study it was treated as clean. The permeability of the particulate 
filter was chosen as $2.5 \times 10^{-9} \mathrm{~m}^{-2}$ to correspond to a pressure drop of $40 \mathrm{~Pa}$ across the particulate filter seen in Smith. ${ }^{(7)}$

The permeability of activated carbon in respirator filters is not well-documented and varies from filter to filter. One estimate for the permeability can be expressed with the help of the Ergun equation, which finds the permeability as a function of the local porosity. ${ }^{(12)}$

The carbon filters are packed using the "snowstorm" filling technique to maximise the packing efficiency. ${ }^{(13)}$ This results in a porosity profile that varies radially throughout the bed, oscillating between high and low porosity near the wall before converging to a uniform porosity near the centre of the bed. This behaviour is described by the empirical Mueller equation ${ }^{(14)}$. The relevant equations describing the porosity distribution and its resultant impact on the permeability of the carbon bed are presented in Appendix 3.

A key parameter in assessing canister performance is the pressure drop across the filter, as increased pressure drop can lead to increased power requirement for the blower or diminished flow rate. The pressure drop was measured by taking the difference of the area-weighted average of the mean pressure values at the inlet and the outlet.

The time until breakthrough of the challenge substance is the other key indicator of canister performance. This is related to the residence time distribution of air in the carbon filter. An estimate for the average residence time, $\tau$, can be found using the air age equation within the canister: $^{(15)}$

$\frac{\partial}{\partial x_{j}}\left(\rho \bar{u}_{j} \tau\right)=\frac{\partial}{\partial x_{j}}\left(\mu_{e f f} \frac{\partial \tau}{\partial x_{j}}\right)+\rho$

where $\rho$ is the gas density, $\mu_{e f f}=\mu_{l}+\mu_{t}$ is the effective viscosity with $\mu_{l}$ and $\mu_{t}$ being the dynamic and turbulent eddy viscosity, respectively. For optimum filter usage, all of the adsorption sites present should be occupied at breakthrough and breakthrough would occur simultaneously across the whole outlet of the carbon filter. It is therefore important to know exactly how much the residence time varies radially across the outlet of the carbon filter. The 
distribution of residence time in the carbon has therefore been analysed at the outlet of the carbon filter, and the mean air age, minimum air age and the standard deviation of air age have been analysed in detail.

\section{Numerical Implementation and Boundary Conditions}

The coupled mass, momentum and age equations are solved in the framework of finitevolume technique using a commercial software called ANSYS Fluent (Release 15.0, Ansys, Inc., Canonsburg, PA). All simulations have been carried out using the second-order upwind discretisation scheme for the advective terms, whereas a second-order central difference scheme is used for the discretisation of the diffusive terms ${ }^{(16)}$. The discretised governing equations are solved using the semi-implicit pressure-velocity coupling enabled by the SIMPLE algorithm. ${ }^{(17)}$ A scaled residual threshold of $10^{-6}$ is considered to be the convergence criteria for all the variables considered here.

The simulations have been conducted under the assumption of axisymmetry based on a geometry considered in the past for practical applications. ${ }^{(7)}$

In many of the simulations, a large zone of flow recirculation was seen close to the outlet after the final corner, causing some reverse flow at the outlet. This is detrimental both to the accuracy, so the geometry was modified to have an extended outlet which would allow the flow to develop. Key characteristics such as the mean pressure drop were still evaluated using true outlet location, rather than the extended simulation outlet.

Although human breathing rates vary considerably, the flow rate was selected based on the most typical case. A constant inlet flowrate of 50L/min was chosen for all simulations, as it represents a breathing rate under intermediate to heavy labour. ${ }^{(10)}$ This equates to a mean velocity $U$ of $1.80 \mathrm{~ms}^{-1}$ at the inlet. Steady-state simulations were used due to the drastic reduction in computational time compared to transient flow, and have previously been shown to give a good indication of canister performance ${ }^{(6,10)}$. Turbulence at the inlet is described by 
two parameters, the turbulence intensity $I=\sqrt{2 k / 3} / U$ and the turbulence integral length scale $l$. A sensitivity study was used to choose $I=10 \%$ and $l=0.07 d$, where $d$ is the inlet diameter. The Neumann boundary condition has been used at the outlet boundary, such that each primitive variable does not change normal to the boundary. All the walls are considered to be no-slip boundaries and thus the velocity components tangential to the wall are considered to zero. The velocity components normal to the walls are also zero due to impenetrability of the solid walls. The boundary conditions for $k$ and $\epsilon$ are discussed in Appendix 1. It was ensured that the mesh used was of sufficient fineness to meet the turbulence model's requirement to solve all equations all the way to the wall, which is discussed in detail in Appendix 1.

\section{Data Analysis}

The impact of changing each parameter was assessed across by carrying out a factorial analysis of variance study, which gives an indication of the significance of each parameter with regard to each response variable, ${ }^{(18)}$ as well as any interaction between parameters. The parameters and levels and the labels used for them are shown in Table 1.

\section{VALIDATION}

Experimental results for pressure drop over a wide range of flow rates across an existing canister filter (shown in Fig. 4) were reported in $\mathrm{Li}^{(6)}$ These results were used to provide validation for the CFD methodology used in this analysis. However, it is worth noting that the geometry shown in Fig.4 is not axisymmetric and thus 3D simulations have been carried out for this configuration. However, the same numerical methodology that used for 2D axisymmetric cases shown in Fig. 3 were employed for 3D simulations in order to compare the pressure drop with respect to the experimental results reported in $\mathrm{Li}^{(6)}$ Two different mesh sizes were used for 3D simulations and mesh independence was verified. 
The boundary conditions used in the 3D simulations were the same as those used for the axisymmetric cases. Two cases were modelled; one in which a fixed permeability was used and one, which used a radial porosity profile, described by the Mueller equation, using a fixed carbon bead size of $0.8 \mathrm{~mm}$. The fixed permeability was found experimentally by measuring the pressure drop throughout both the filter paper and the carbon bed independently and fitting them to the Darcy equation.

The pressure drop across the canister was measured at 50L/min, and is shown in Table A3 in Appendix 2. Both the fixed permeability and the Mueller porosity profile slightly underpredict the experimental results but come close enough to suggest that this methodology gives a good indicator of the actual performance, with the Mueller equation giving the more accurate prediction. There is a small difference between the results found here and the results seen in Li. ${ }^{(6)}$ This minor difference arises due to the fact that a small screw in the canister geometry has not been accounted for the current simulations.

Typically, an axisymmetric model takes of the order of one hour to converge on the computational hardware given in Table A3 in Appendix 2, in contrast to approximately seven hours for the 3D simulations. For this reason, axisymmetric simulations were favoured when a large number of variations to the geometry were desired.

\section{RESULTS AND DISCUSSION}

\section{General Flow Features}

In general, a large pressure gradient has been observed throughout the porous media in all cases, with a very small pressure gradient throughout the empty canister. The pressure profile seen throughout the carbon bed depends largely on whether or not the carbon backs directly onto the canister back wall, or if a space is left between the carbon and the canister wall. 
As shown in Fig. 5, in cases where there is a space between the carbon outlet and the canister wall, the pressure gradient is mostly uniform in the axial direction and there is very little variation in radial pressure throughout the carbon bed. In contrast to this, in Fig. 6 it is clear that the pressure varies both radial and axial directions throughout the carbon bed, as the flow against the back wall is directed inwards towards the outlet. This also results in a significantly increased pressure drop in these cases.

The air age distribution in the carbon bed was significantly affected by the porosity profile of the carbon bed. In all cases close to the canister wall, an area of much lower residence time could be seen in the area with the highest porosity, as this region offers weaker resistance to flow so air passes through more rapidly. The velocity close to the canister wall is indicative of the porosity profile, with alternating regions of high and low porosity as shown in Fig. 7, with higher velocities seen at radial distances where the porosity is low.

Similar to the mean pressure difference, the air age profile within the carbon bed depends significantly on whether or not the carbon bed backs directly onto the canister back wall. In cases where it does not, the air age increase is more uniform in the axial direction away from the canister wall as shown in Fig. 8. When the carbon bed backs directly onto the wall there is more flow in the radial direction, which creates the air age distribution shown in Fig. 9.

\section{Summary of Effects of Geometry Changes}

The full effects of each parameter on each of the measured variables are described in more detail in the "Analysis of Variance" section. The following is a summary of the major trends.

\section{Space between Carbon Bed and Canister Wall}

Fig. 10 shows that the cases in which the carbon bed backs directly on to the wall have a far larger pressure drop than all other cases, typically by a factor of three. This effect is somewhat diminished when the inner angle of the canister towards the outlet is also curved. The $2 \mathrm{~mm}$ rear spacing reduces the pressure drop by a factor of three in most cases, and the $6 \mathrm{~mm}$ rear 
spacing by a further 5-25\%. Introducing bracketing into the rear gap slightly increases the pressure drop, typically in the range of $10-50 \mathrm{~Pa}$ compared to the $6 \mathrm{~mm}$ rear spacing, with the larger spaces between bracket holes generally causing a slightly larger pressure drop.

As Fig. 11 shows, the cases, where the carbon bed backs directly onto the canister wall, have a much larger mean residence time, ranging from $50 \%$ to $100 \%$ larger than all other cases. However, this does not provide a substantial benefit, as Fig. 12 shows that the minimum residence time is reduced by up to $50 \%$.

\section{Curving the Canister Wall}

Curving the outside of the canister wall typically provided a small reduction (5\% to 20\%) in mean residence time (see Fig. 11), although this was possibly due to the reduction in carbon volume. It also provided a small increase (up to 20\%) in the minimum mean residence time (see Fig. 12).

Curving the inside corner of the canister wall decreased the pressure drop and increased the mean and minimum residence times only in cases where the carbon bed backed directly onto the canister wall.

\section{Bracketing between Filter Layers}

\section{Analysis of Variance Results}

Typically, both sizes of bracket caused a very small increase (20Pa to $70 \mathrm{~Pa}$ ) in pressure drop. This was larger for the finer bracket as demonstrated in Fig. 10.

\section{Pressure Drop}

The ANOVA results shown in Table 2 indicate that all factors have a significant impact on the mean pressure drop. By far the greatest factor influencing the mean pressure drop is the spacing at the rear of the carbon bed, as shown in Fig. 10. In cases, where the carbon bed backs directly onto the rear wall of the canister, the pressure drop is typically far higher than in cases 
that have a space between the carbon and the wall, typically by a factor of three. A modest decrease (of the order of $10 \%$ ) in the mean pressure drop is seen increasing the length of this space from $2 \mathrm{~mm}$ to $6 \mathrm{~mm}$, and an increase of approximately $10 \mathrm{~Pa}$ to $50 \mathrm{~Pa}$ in pressure drop was seen if that $6 \mathrm{~mm}$ gap featured either shape of bracketing.

There is a two-factor interaction between the shape of the inner canister curve and the gap at the rear of the carbon. Fig. 10 shows that this interaction is only significant in the cases which have no rear gap. The curve means that less of the carbon backs directly onto the carbon wall than the configuration where the back of the canister is straight. The impact of curving the outside edge of the canister on the mean pressure drop was found to be extremely small.

Bracketing between the particulate filter and carbon bed had some impact on the mean pressure drop. Applying a $6 \mathrm{~mm}$ spaced bracket increased the mean pressure drop by an average of $38 \mathrm{~Pa}$ over the un-bracketed cases. Applying a 3mm spaced bracket increased the pressure drop by an average of 63Pa over the un-bracketed cases.

\section{Mean Air Age at rear of Carbon Bed}

The ANOVA results shown in Table 3 show that all factors have a significant impact on the mean residence time at the outlet of the carbon. As with pressure drop, by far the greatest factor influencing the mean air age at the carbon outlet is the spacing at the rear of the carbon. In cases where the carbon backs directly onto the rear wall of the canister the mean air age at the carbon outlet is typically $50 \%$ higher than in cases that have a space between the carbon and the wall, as can be seen from Fig. 11.

Curving the outer canister wall generally had a small reduction on the mean residence time, although this was likely to be largely due to the associated reduction in carbon volume, on average resulting in a mean residence time reduced approximately by $5 \%$. 
Bracketing between the particulate filter and carbon bed had a very small impact on the mean residence time at the carbon outlet. The $3 \mathrm{~mm}$ spaced bracket on average increased mean residence time by $2.5 \%$, and the $6 \mathrm{~mm}$ bracket on average increased residence time by $5 \%$.

There is a significant two-factor interaction between the shape of the inner canister curve and the gap at the rear of the carbon. The mean air age at the rear of the carbon bed significantly increases in cases where the carbon bed backs onto the canister wall when it is curved at the outlet. As less of the carbon backs directly onto the curved canister wall, the mean air age assumes values similar to those in cases where there is a space between the rear wall and the carbon bed.

\section{Minimum air age at rear of carbon bed}

The most important factor affecting the minimum mean air age at the rear of the canister was the presence of a gap or bracket at the rear of the carbon, as shown in Fig. 12. Bracketing towards the outside of the canister had a significant effect in increasing the minimum residence time identified, typically by $10 \%$ to $50 \%$. Because of the porosity profile towards the canister wall, there is an area of very low porosity approximately one particle diameter from the canister wall, where the minimum air age is likely to be found.

Curving the outer canister wall generally caused a modest increase of the minimum residence time, possibly due to the increased length of the canister wall, causing flow in the porous zone close to the wall to take a slightly longer path. On average, this increased the minimum mean residence time approximately by $10 \%$.

\section{CONCLUSION}

Sixty steady-state axisymmetric CFD simulations have been carried out to assess the effects of small alterations to the geometry of a representative CBRN canister, with respect to the mean pressure drop and the mean residence time distribution throughout the canister. The cases in which the carbon filter backs directly onto the canister rear wall have a much larger pressure 
drop than all other cases, typically by a factor of three. This is due to the longer route through the porous medium the flow must typically take, as flow close to the cylinder wall will reach the rear wall and then have to flow inwards radially to reach the outlet. Increasing the distance travelled by the air through the porous medium results in an increased pressure drop. This inward flow will also drive air entering the carbon bed closer to the canister axis more rapidly towards the outlet of the carbon bed, reducing the minimum air age observed. These cases exhibit the largest variation of mean air age, and rapid breakthrough of the filter is likely to occur for such a low minimum air age. This suggests that the mean air age alone at the carbon outlet is not a good indicator of filter performance. Moreover, the simulations show the carbon filter backing directly onto the canister rear wall is highly detrimental to the overall performance.

Bracketing in the space between the carbon and the canister rear wall has a large impact on the minimum residence time, significantly reducing it in nearly all cases. Both bracketing types have a similar impact; the most important requirement is that the region of highest porosity close to the wall is adequately blocked by the bracket. The bracketing used in axisymmetric simulations cannot be entirely representative of real 3D brackets. There is a possibility that in three dimensions some brackets may cause behaviour similar to that seen in cases where the carbon backs directly onto the wall, causing flow in the radial direction, which may be detrimental to performance.

Changing the curvature at the outer wall may help to direct the flow through the most porous region to take a longer path and improve the minimum air age, with a very small impact on the mean air age and mean pressure drop.

Although it is impossible for axisymmetric simulations to capture all of the features of a full 3D canister geometry, certain flow features such as the alternating regions of high and low porosity close to the wall (and the ensuing velocity) should remain consistent between two and 
three dimensions. Axisymmetric CFD modelling of adsorption through a simplified canister geometry has previously given a good match to experimental results. ${ }^{(7)}$ The results of an axisymmetric study can therefore be used as a basis from which to inform some aspects of 3D canister design.

It is worth noting the present analysis focuses on steady-state simulations for a given flow rate but the qualitative nature of the present findings remains unaltered for steady flow rates within from $10 \mathrm{~L} / \mathrm{min}$ to $150 \mathrm{~L} / \mathrm{min}$. However, in breath responsive PAPRs flow through the filters remains unsteady and the filter performance for transient flow is yet to be analysed. Furthermore, extensive analysis based on 3D simulations for both steady and unsteady flows will be needed for accurate quantitative predictions. Some of the aforementioned issues will form the basis of further investigations.

\section{ACKNOWLEDGMENTS}

The authors are grateful to EPSRC, UK for financial support.

\section{REFERENCES}

1 Klockseth, M.O.: Breath responsive powered air purifying respirator apparatus. USA: Interspiro $\mathrm{Ab}, 2008$.

2 Gardner, P.D.: Multi-agent end-of-service-life indicator for respirator filters. USA: The United States Of America As Represented By The Secretary Of The Army, 2008.

3 Ambesi, D., R. Bouma, E. den Hartog, and C.R. Kleijn: Predicting the chemical protection factor of CBRN protective garments. Journal of occupational and environmental hygiene 10(5): 270-276 (2013).

4 Lücke, T., and H. Fissan: The prediction of filtration performance of high efficiency gas filter elements. Chemical Engineering Science 51(8): 1199-1208 (1996).

5 Chen, D.-R., D.Y.H. Pui, and B.Y.H. Liu: Optimization of pleated filter designs using a finiteelement numerical model. Aerosol Science and Technology 23(4): 579-590 (1995).

6 Li, C.: Aerodynamic behaviour of a gas mask canister containing two porous media. Chemical Engineering Science (64): 1832-1843 (2008).

7 Smith, A.G., S. MW, and K. Taylor: Prediction of heat and Mass Transfer in Canister Filters. PHOENICS User Conference (2004).

8 Su, Y.-C., and C.-C. Li: Computational fluid dynamics simulations and tests for improving industrial-grade gas mask canisters. Advances in Mechanical Engineering 7(8): 1687814015596297 (2015).

9 Standard Respirator Testing Procedures: Air-Purifying Respirators with Chemical, Biological, Radiological, and Nuclear Protection: NIOSH, 2016. 
10 Suzin, Y., I. Nir, and D. Kaplan: The effect of flow pattern on adsorption of dimethyl methyl phosphonate in activated carbon beds and canisters. Carbon 38(8): 1129-1133 (2000).

11 Wilcox, D.C.: Turbulence Modeling for CFD. In Turbulence Modeling for CFD. California: DCW Industries, Inc., 1994.

12 Ergun, S.: Flow through packed columns. Chemical Engineering Progress 48: 89-94 (1952).

13 Harris, E.: Improvements in or relating to the filling of granular materials into containers. United Kingdom, 1946.

14 Mueller, G.E.: Radial void fraction distributions in randomly packed fixed beds of uniformly sized spheres in cylindrical containers. Powder Technology 72(3): 269-275 (1992).

15 Baléo, J.-N., and P.L. Cloirec: Numerical simulation of the spatial distribution of mean residence time in complex flows through porous media. Progress of Theoretical Physics Supplement 138: 690695 (2000).

16 Launder, B.E., and B.I. Sharmer: Application of the Energy-Dissipation Model of Turbulence to the Calculation of Flow Near a Spinning Disc. Letters in Heat and Mass Transfer 1(2): 131-138 (1974). 17 Patankar, S.: Numerical Heat Transfer and Fluid Flow: Hemisphere Publishing Corportation, 1980.

18 Mann, P.S., and C.J. Lacke: Introductory Statistics. Hoboken: Wiley, 2001.

19 Andrade Jr, J.S., U.M.S. Costa, M.P. Almeida, H.A. Makse, and H.E. Stanley: Inertial effects on fluid flow through disordered porous media. Physical Review Letters 82(26): 5249 (1999). 


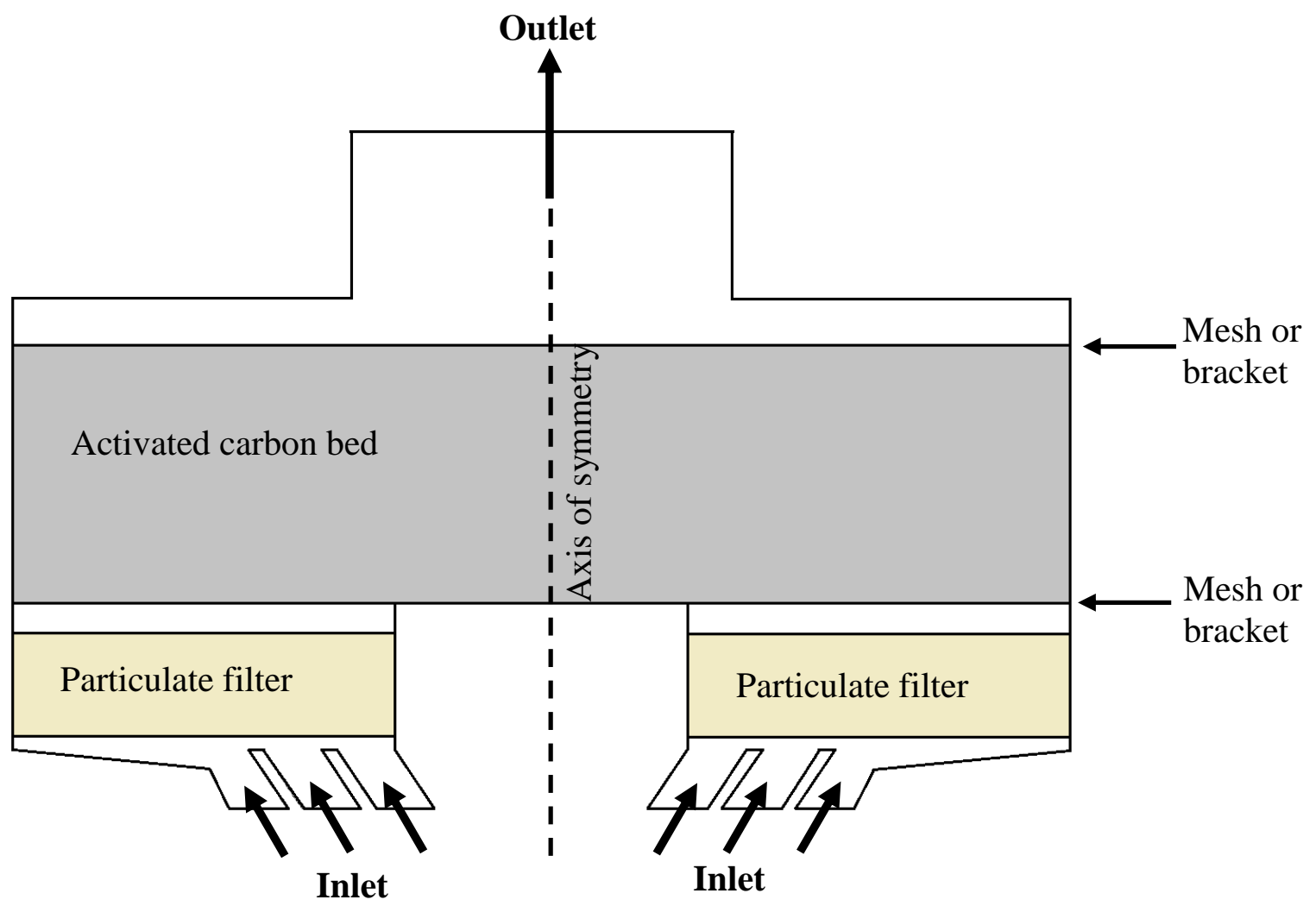

Figure 1: Cross section of a typical CBRN filter design. 


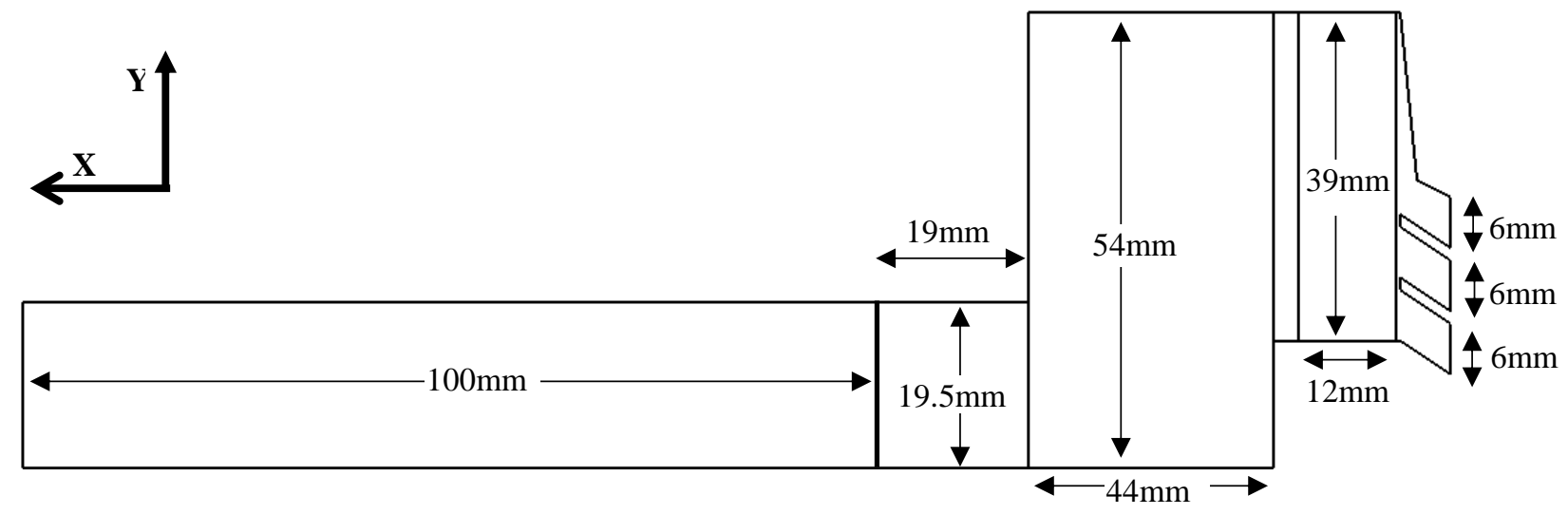

Figure 2. Schematic diagram of the computational domain for the baseline geometry. 

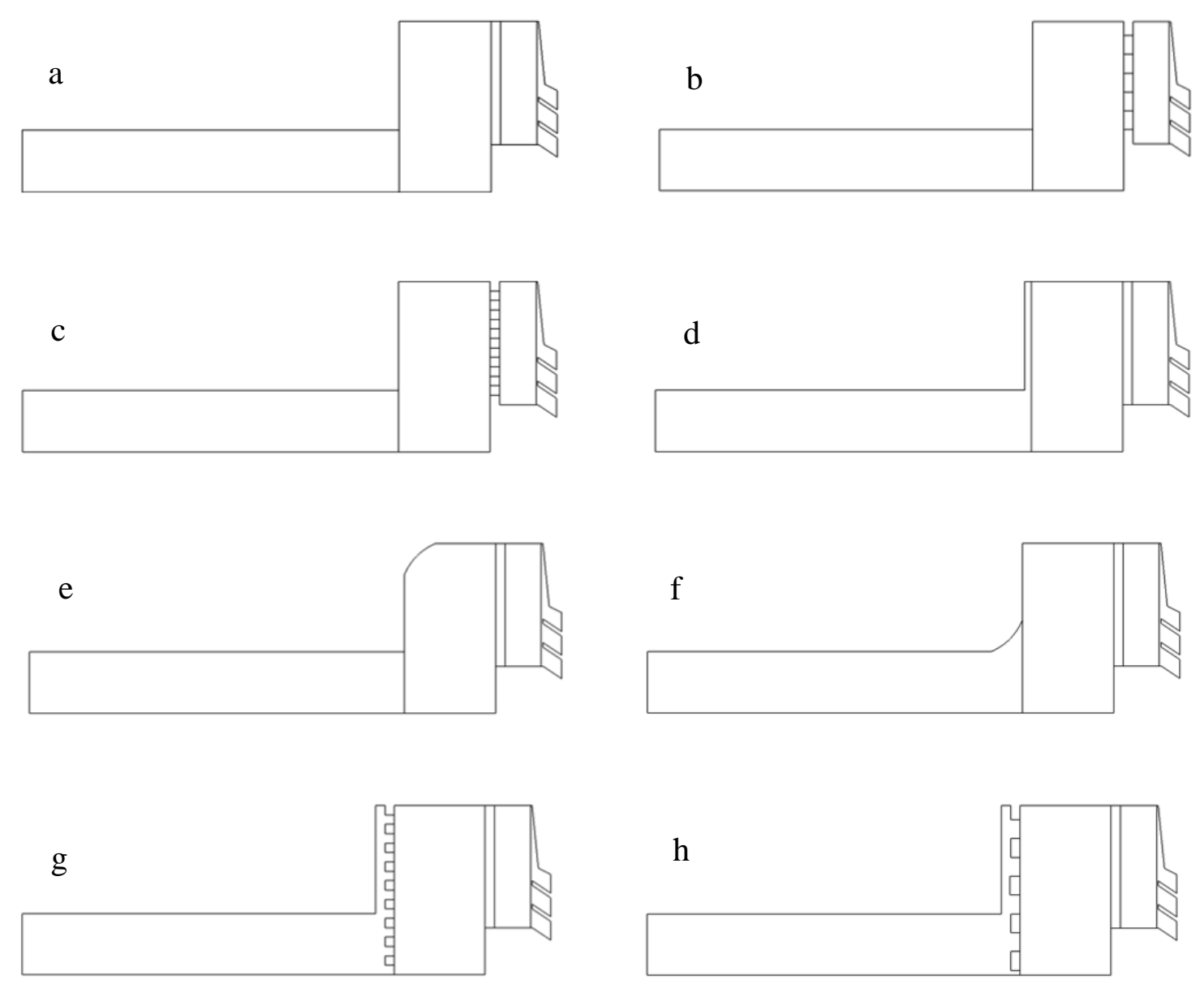

Figure 3. Summary of the variations of the canister geometry considered here: the base case (3a), two sizes of bracketing before the carbon filter ( $3 \mathrm{~b}$ and $3 \mathrm{c}$ ), spacing between the carbon filter and the canister wall (3d), curvature of the canister outer wall ( $3 \mathrm{e}$ and $3 \mathrm{f}$ ) and two different sizes of bracketing after the carbon filter ( $3 \mathrm{~g}$ and $3 \mathrm{~h}$ ) 


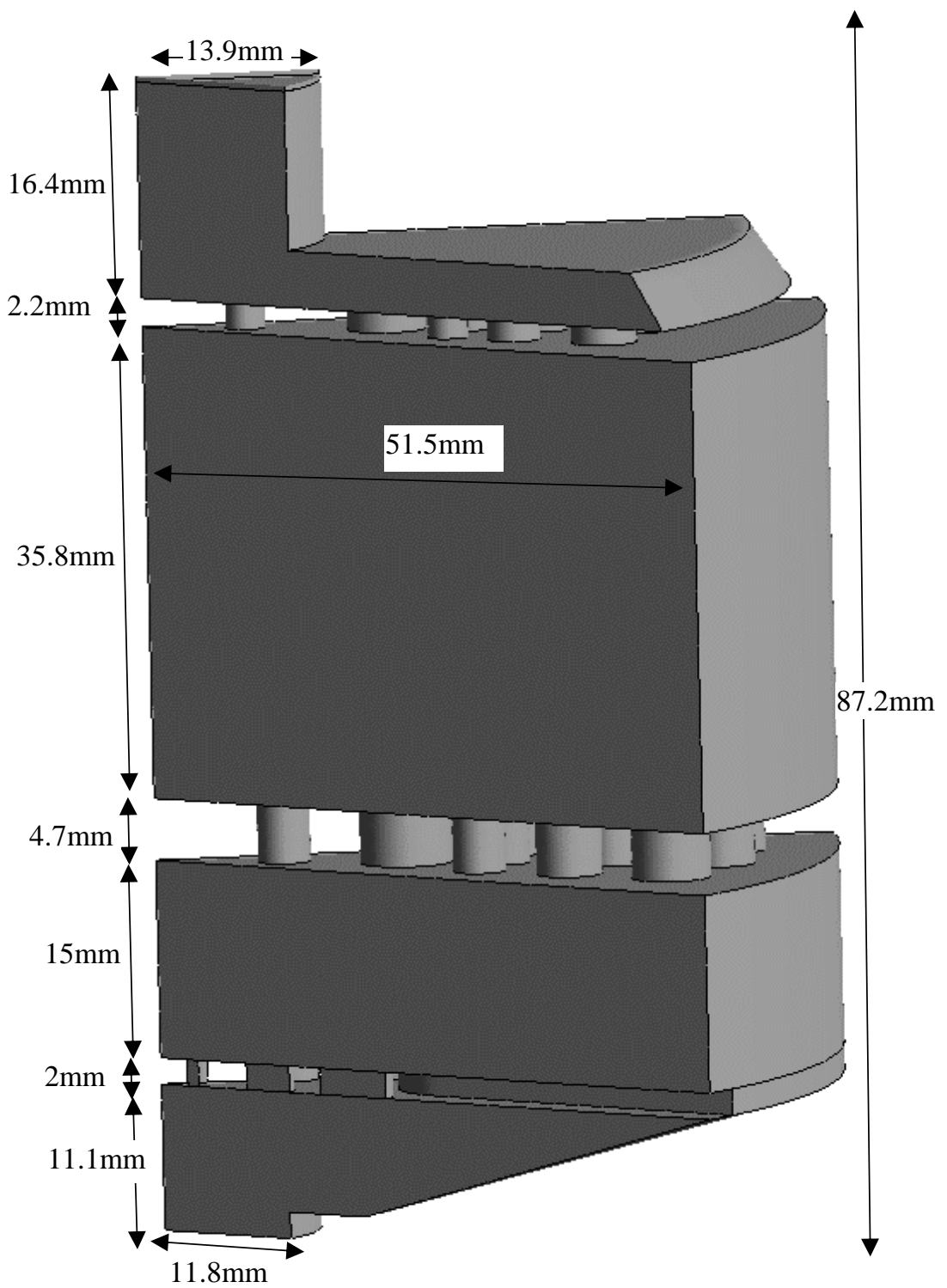

Figure 4. Geometric configuration of canister modelled for 3D simulation. 


\section{Pressure Difference from Outlet $(\mathbf{P a})$}

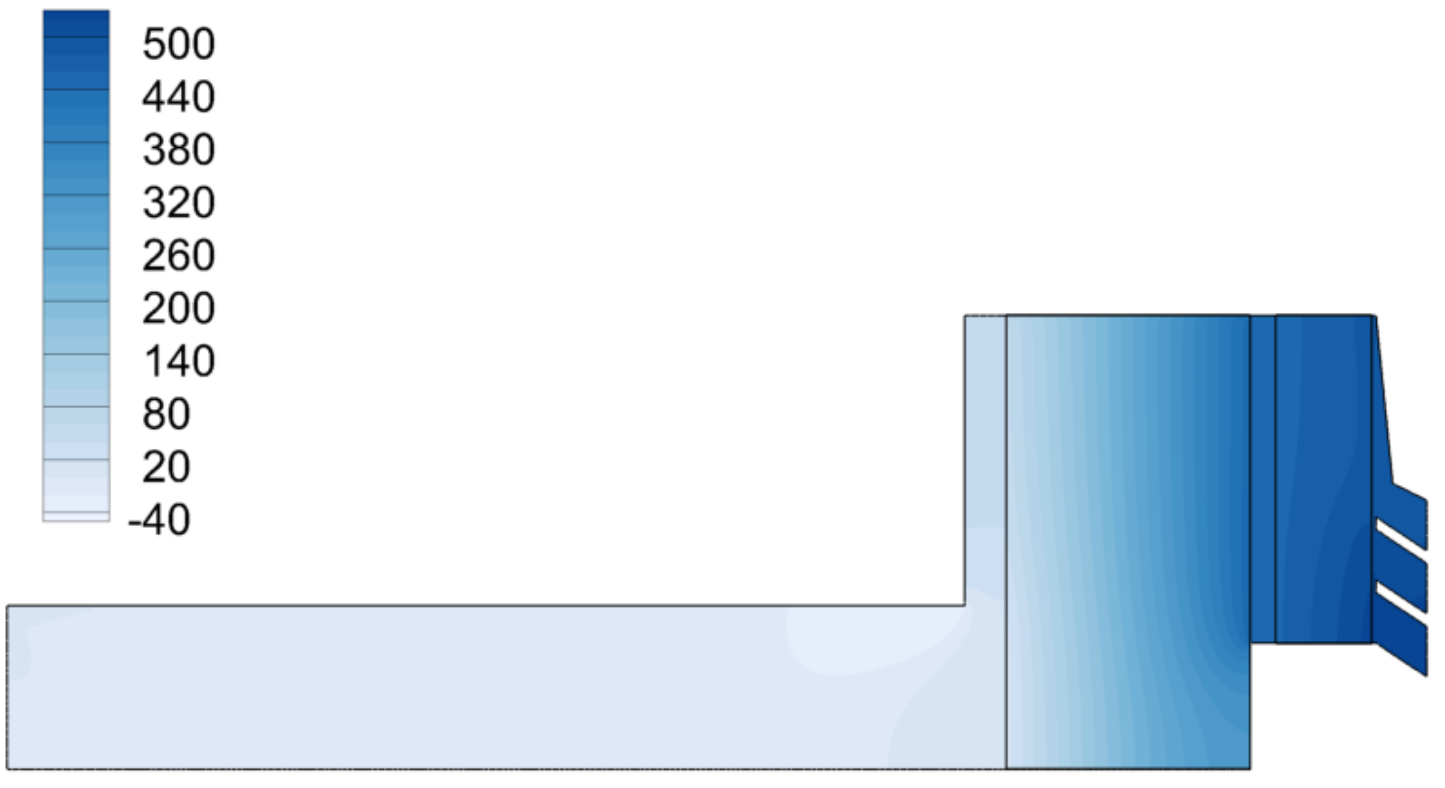

Figure 5. Distribution of pressure difference from the outlet in the case with curvature towards the outlet and extended spacing between the carbon bed and the canister rear wall (the case with the lowest pressure drop). 
Pressure Difference from Outlet (Pa)

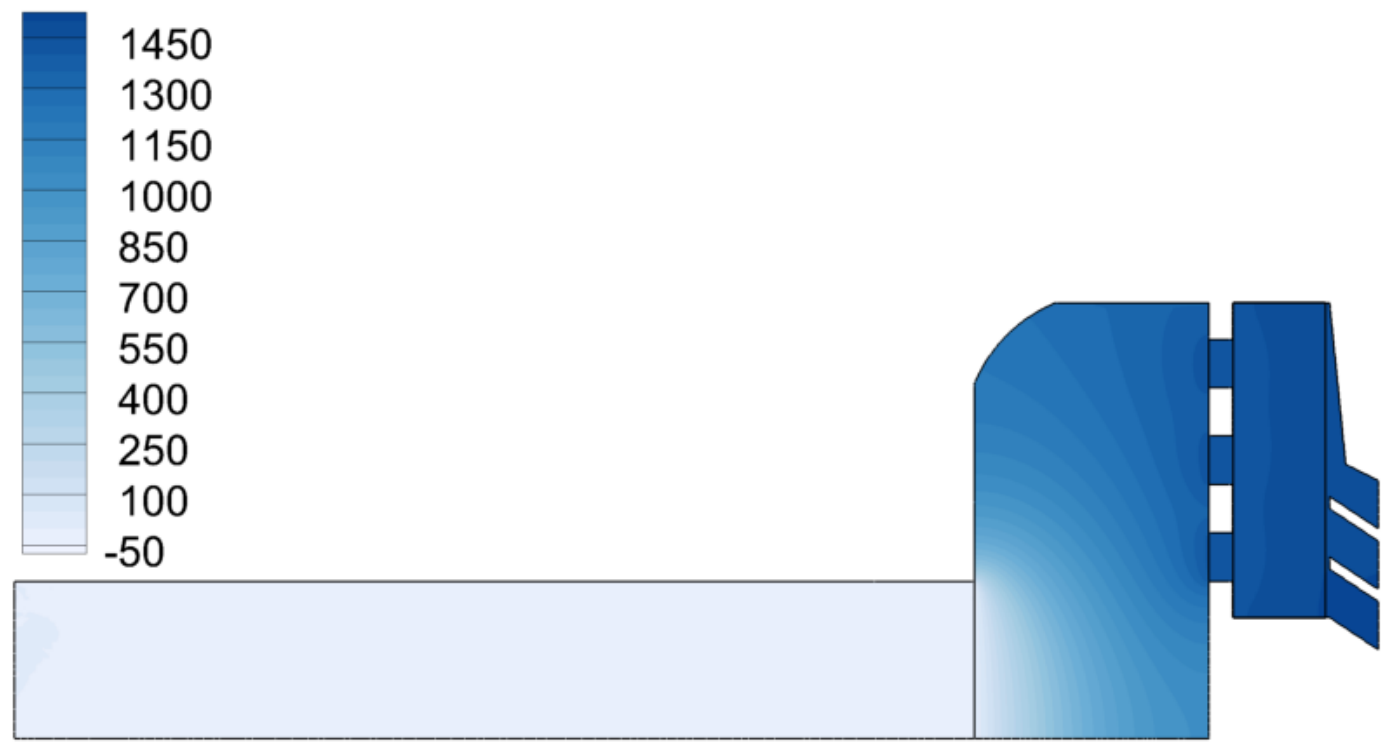

Figure 6. Distribution of pressure difference from the outlet in the case with the bracketing between the carbon and particulate filters and curvature of the outer wall (the case with the greatest pressure drop. 


\section{Axial Velocity $(\mathbf{m} / \mathbf{s})$}

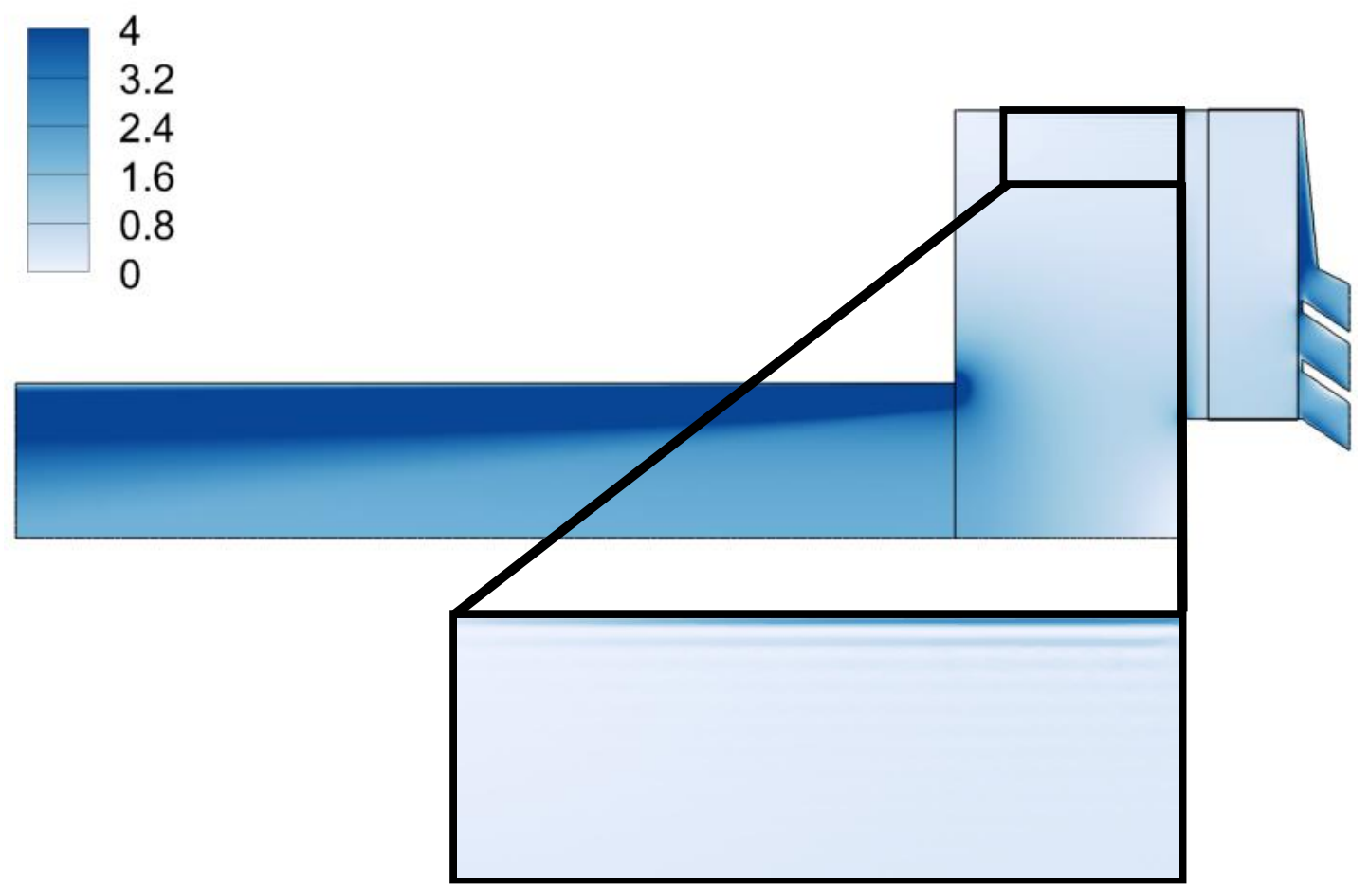

Figure 7. Alternating regions of high and low velocity close to the canister wall in the base geometry. 


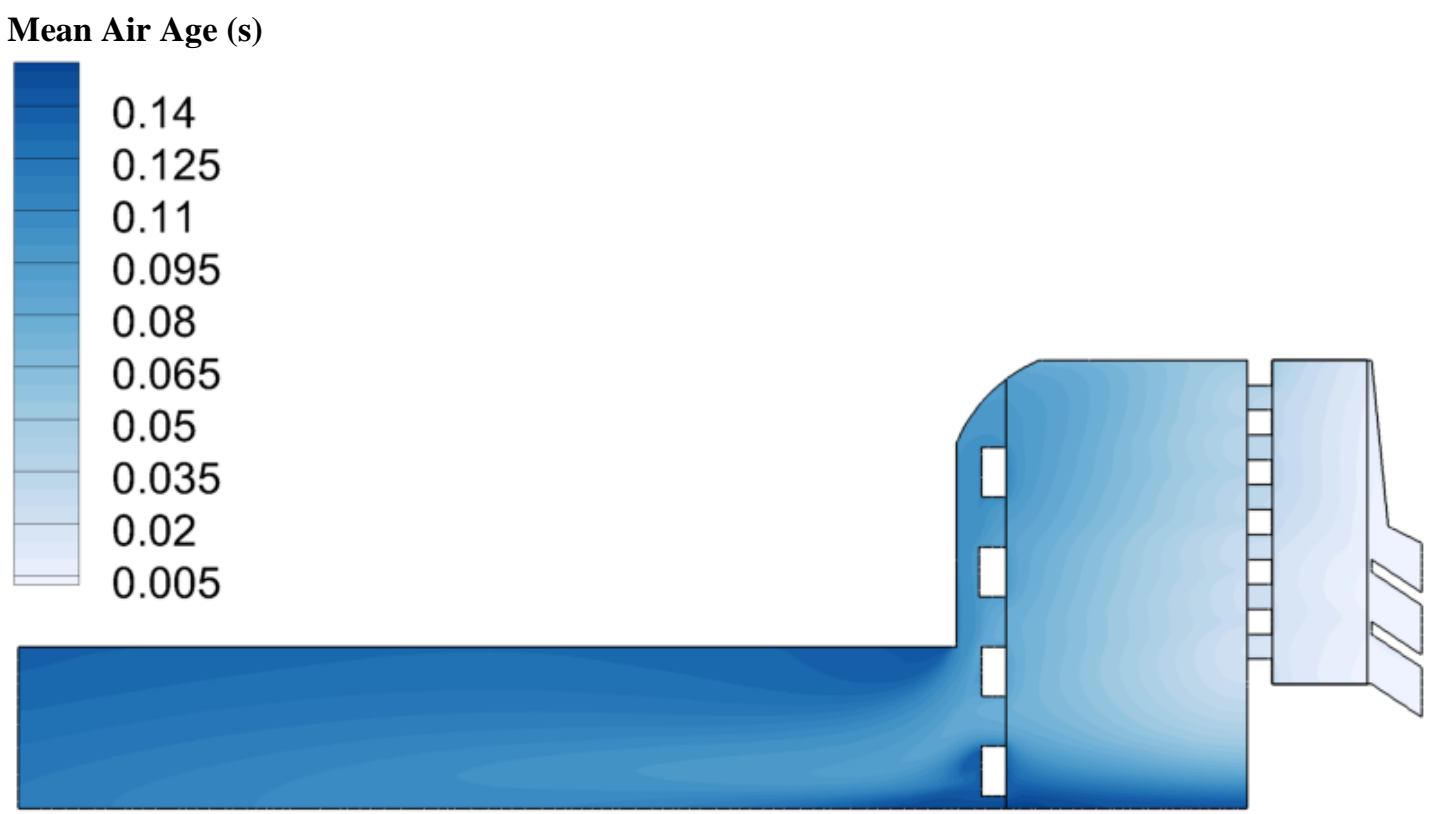

Figure 8. Mean age distribution throughout the canister in the case with a curved outer canister wall and bracketing between the carbon and particulate filter and bracketing between the carbon and the rear wall (the case with the smallest mean air age at the carbon outlet).

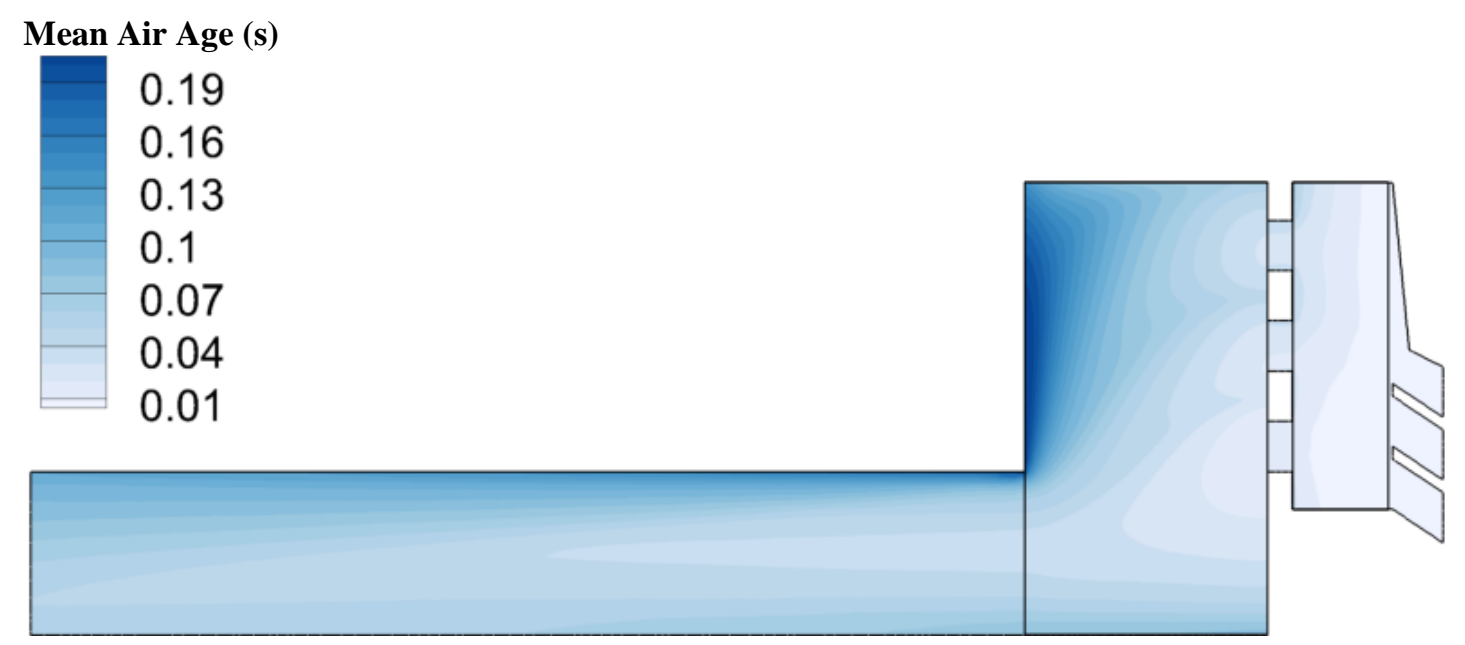

Figure 9. Mean air age distributionthroughout the canister in the case with bracketing between the particulate filter and carbon bed (the case with the greatest mean air age at the carbon outlet). 


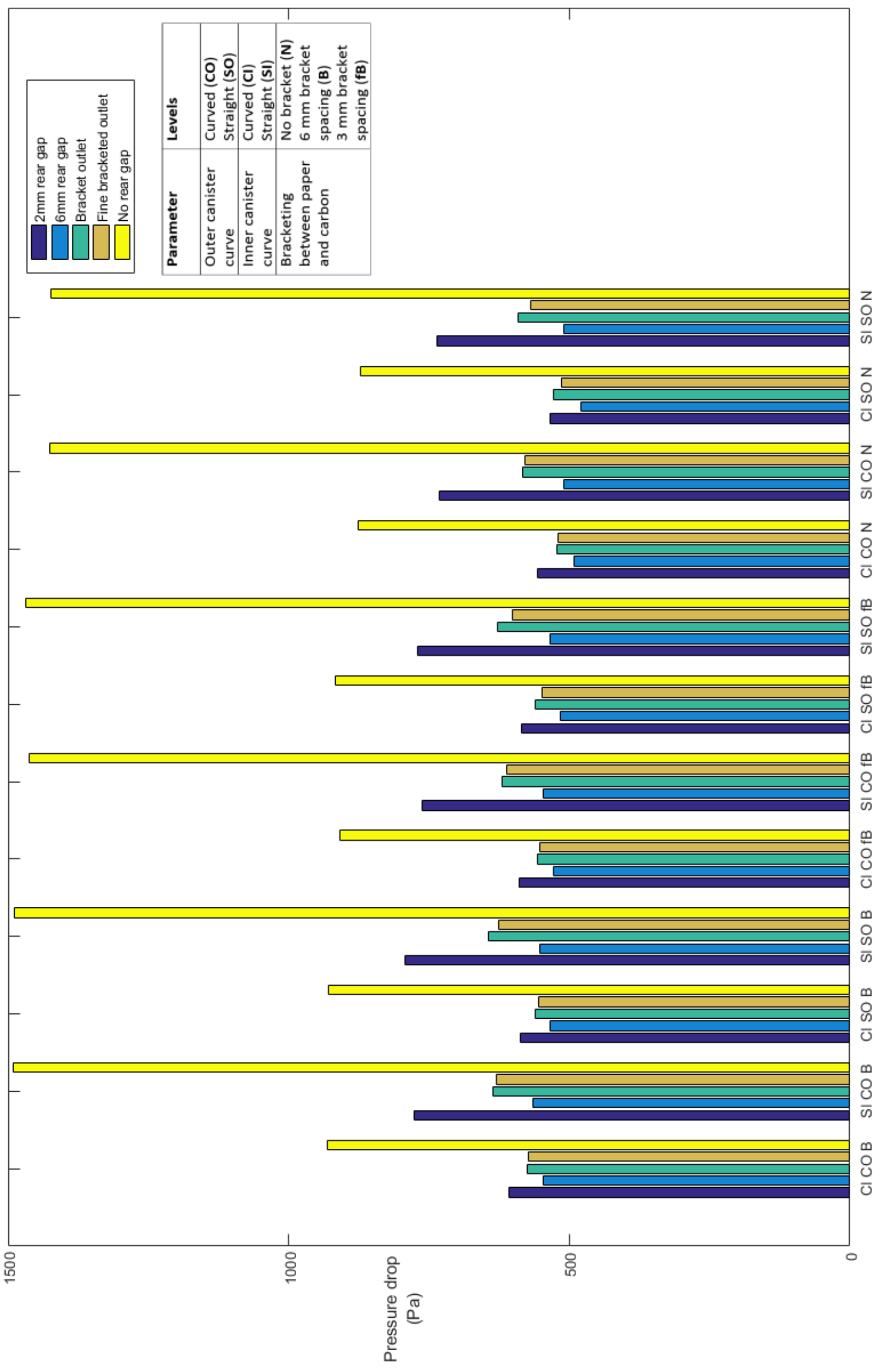

Figure 10. Pressure drop throughout the canister for all canister geometries. 


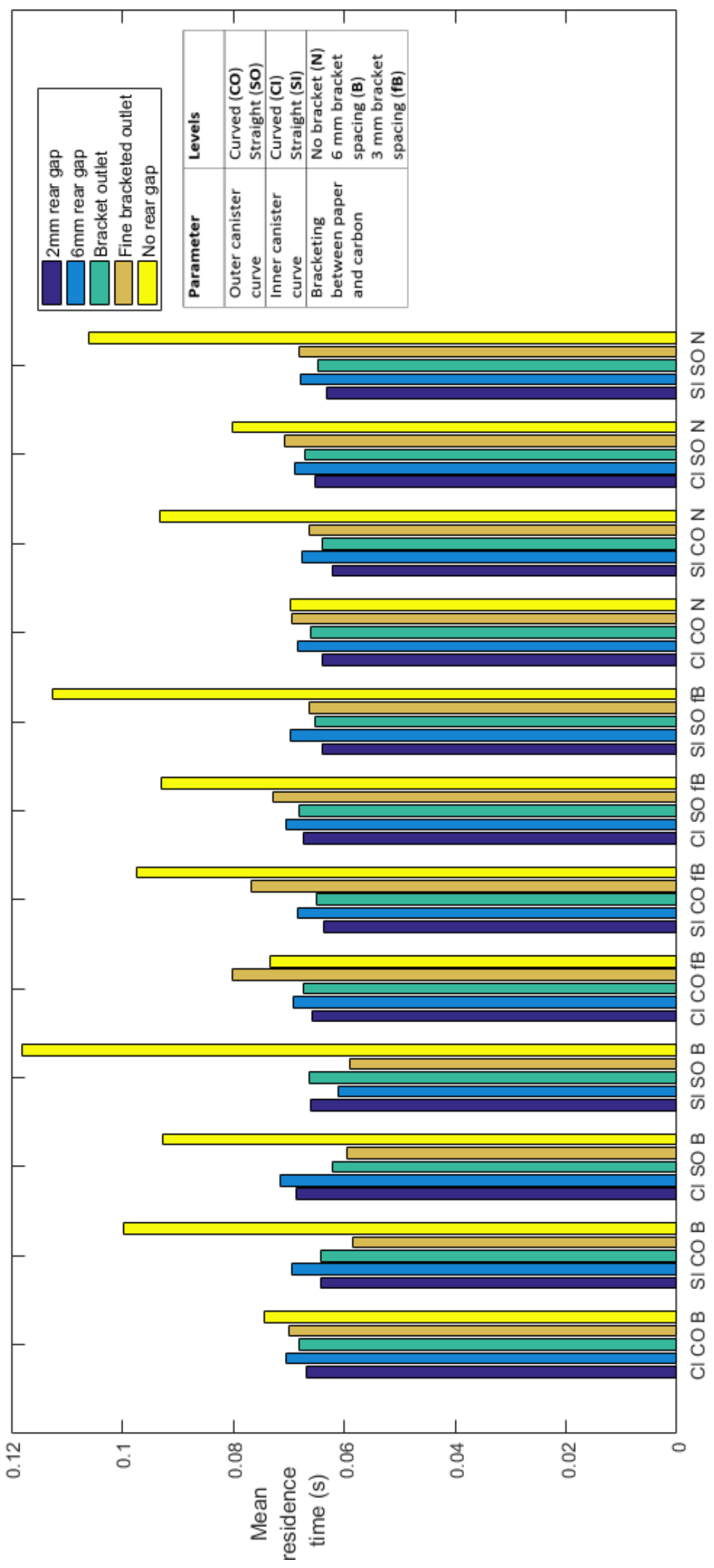

Figure 11. Mean residence time at the rear of the carbon bed for all canister geometries. 


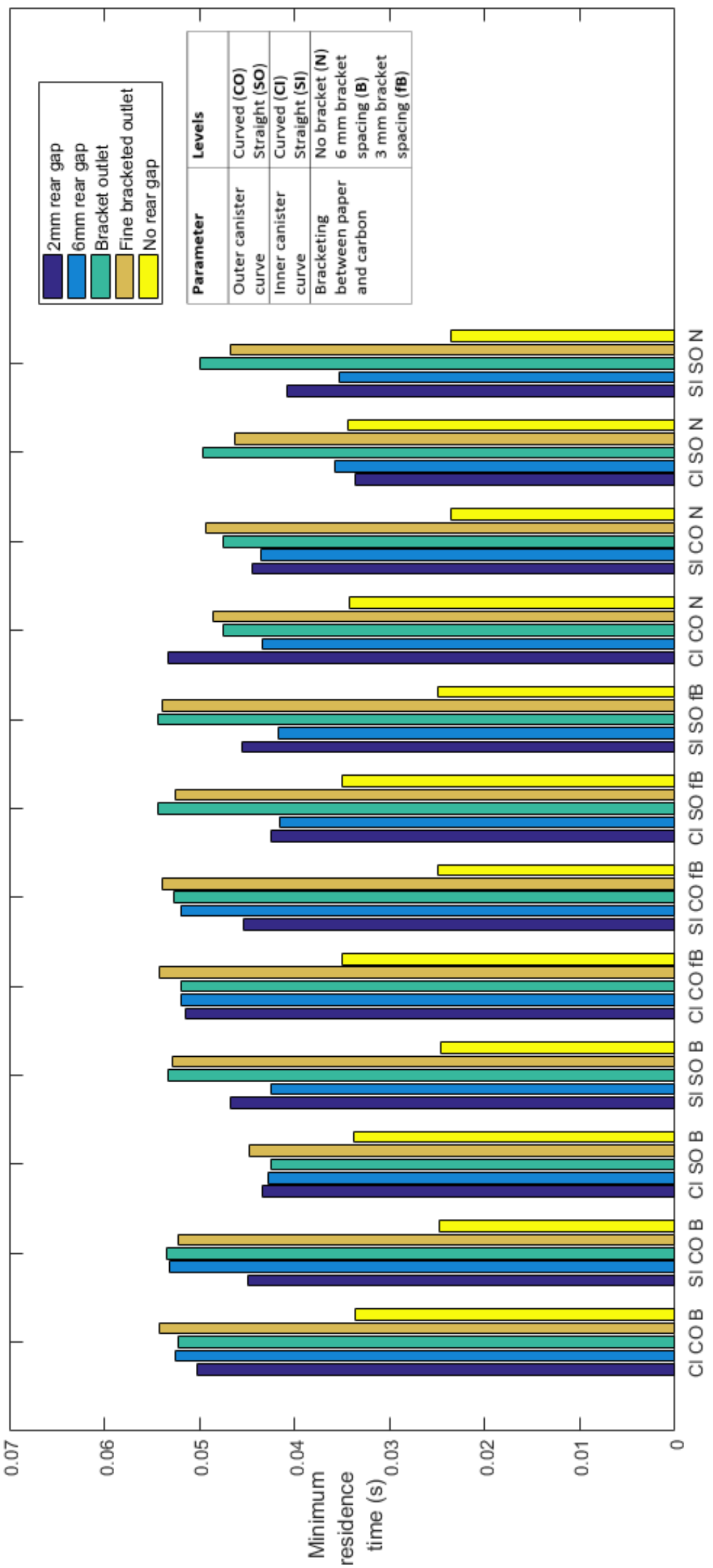

Figure 12. Minimum mean residence at the rear of the carbon bed for all canister geometries. 


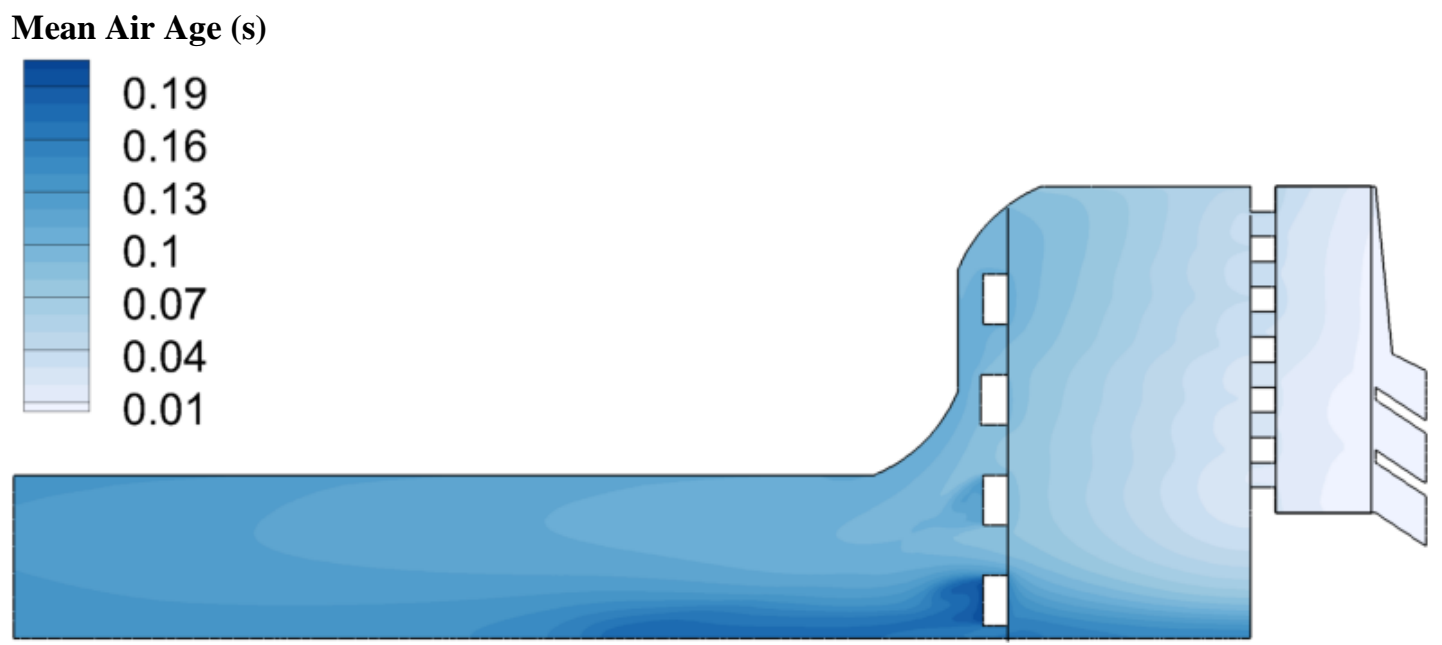

Figure 13. Distribution of mean air age throughout the canister in the case with the largest minimum residence time at the rear of the carbon bed.

\section{Mean Air Age (s)}

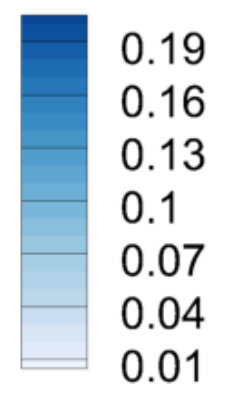

0.16

0.13

0.1

0.01

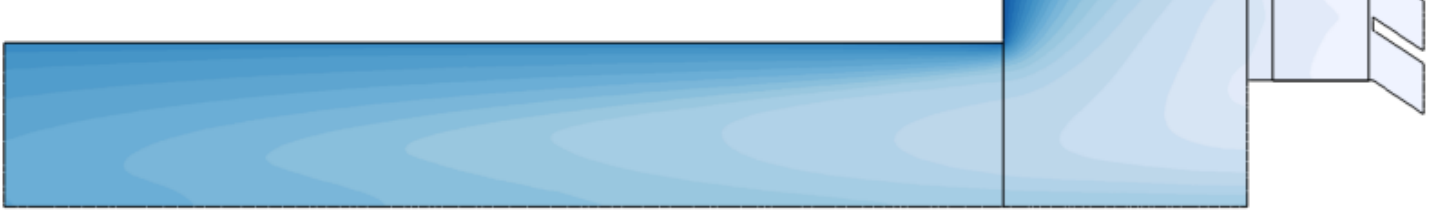

Figure 14. Distribution of mean air age throughout the canister in the case with the smallest minimum residence time at the rear of the carbon bed. 


\section{Figure Captions}

Figure 1. Cross section of a typical CBRN filter design.

Figure 2. Schematic diagram of the baseline geometry of the computational domain.

Figure 3. Summary of the variations of the canister geometry considered here: the base case (5a), two sizes of bracketing before the carbon filter ( $5 b$ and $5 c$ ), spacing between the carbon filter and the canister wall (5d), curvature of the canister outer wall (5e and 5f) and two different sizes of bracketing after the carbon filter $(5 \mathrm{~g}$ and $5 \mathrm{~h})$.

Figure 4. Geometric configuration of canister modelled in 3D simulations.

Figure 5. Distribution of pressure difference from the outlet in the case with curvature towards the outlet and extended spacing between the carbon bed and the canister rear wall (the case with the lowest pressure drop).

Figure 6. Distribution of pressure difference from the outlet in the case with the bracketing between the carbon and particulate filters and curvature of the outer wall (the case with the greatest pressure drop).

Figure 7. Alternating regions of high and low velocity close to the canister wall in the base geometry.

Figure 8. Mean age distribution throughout the canister in the case with a curved outer canister wall and bracketing between the carbon and particulate filter and bracketing between the carbon and the rear wall (the case with the smallest mean air age at the carbon outlet.

Figure 9. Mean air age distributionthroughout the canister in the case with bracketing between the particulate filter and carbon bed (the case with the greatest mean air age at the carbon outlet).

Figure 10. Pressure drop throughout the canister for all canister geometries.

Figure 11. Mean residence time at the rear of the carbon bed for all canister geometries.

Figure 12. Minimum mean residence at the rear of the carbon bed for all canister geometries. Figure 13. Distribution of mean air age throughout the canister in the case with the largest minimum residence time at the rear of the carbon bed.

Figure 14. Distribution of mean air age throughout the canister in the case with the smallest minimum residence time at the rear of the carbon bed. 
Table 1. Geometric parameters varied and their respective levels used in the analysis of variance study.

\begin{tabular}{ll}
\hline Parameter & Levels \\
\hline Outer canister curve & Curved (CO), Straight (SO) \\
Inner canister curve & Curved (CI), Straight (SI) \\
Spacing at rear of carbon & $\begin{array}{l}\text { No gap, 2 mm gap, 6 mm gap, 6 mm bracket } \\
\text { spacing in 6mm gap, } 3 \mathrm{~mm} \text { bracket spacing } \\
\text { in } 6 \mathrm{~mm} \text { gap }\end{array}$ \\
& $\begin{array}{l}\text { No bracket (N), 6 mm bracket spacing (B), } 3 \\
\text { mm bracket spacing (fB) }\end{array}$ \\
\hline
\end{tabular}

Table 2. Results of the ANOVA analysis with respect to pressure drop.

\begin{tabular}{llll}
\hline Source & $\begin{array}{l}\text { Degrees } \\
\text { freedom }\end{array}$ & F & Significance \\
\hline CI & 1 & 18284 & 0 \\
CO & 1 & 9 & 0.0055 \\
Inlet bracket & 2 & 561 & 0 \\
Rear gap & 4 & 34184 & 0 \\
CI*CO & 1 & 10 & 0.004 \\
CI*Inlet bracket & 2 & 2 & 0.1668 \\
CI* Rear gap & 4 & 5618 & 0 \\
CO*Inlet & 2 & 1 & 0.2793 \\
bracket & & & \\
CO*Rear gap & 4 & 4 & 0.0082 \\
Inlet*Rear gap & 8 & 1 & 0.3451 \\
Error & 30 & & \\
\hline
\end{tabular}

Table 3. Results of the ANOVA analysis with respect to the mean residence time in the carbon at the rear of the carbon bed.

\begin{tabular}{llll}
\hline Source & $\begin{array}{l}\text { Degrees } \\
\text { freedom }\end{array}$ & F & Significance \\
\hline CI & 1 & 14.88 & 0.0006 \\
CO & 1 & 13.27 & 0.001 \\
Inlet bracket & 2 & 8.27 & 0.0014 \\
Rear gap & 4 & 240.3 & 0 \\
CI*CO & 1 & 0.01 & 0.9292 \\
CI*Inlet bracket & 2 & 0.36 & 0.7031 \\
CI* Rear gap & 4 & 64.8 & 0 \\
CO*Inlet & 2 & 0.29 & 0.7474 \\
bracket & & & \\
CO*Rear gap & 4 & 26.72 & 0 \\
Inlet*Rear gap & 8 & 7.38 & 0 \\
Error & 30 & & \\
\hline
\end{tabular}


Table 4. Results of the ANOVA analysis with respect to the minimum residence time in the carbon at the rear of the carbon bed.

\begin{tabular}{llll}
\hline Source & $\begin{array}{l}\text { Degrees } \\
\text { freedom }\end{array}$ & F & Significance \\
\hline CI & 1 & 4.77 & 0.037 \\
CO & 1 & 29.97 & 0 \\
Inlet bracket & 2 & 15.68 & 0 \\
Rear gap & 4 & 135.89 & 0 \\
CI*CO & 1 & 6.9 & 0.0134 \\
CI*Inlet bracket & 2 & 0.99 & 0.3846 \\
CI* Rear gap & 4 & 10.58 & 0 \\
CO*Inlet & 2 & 0.6 & 0.5576 \\
bracket & & & \\
CO*Rear gap & 4 & 7.5 & 0.0003 \\
Inlet*Rear gap & 8 & 1.77 & 0.124 \\
Error & 30 & & \\
\hline
\end{tabular}




\section{Table Captions}

Table 1. Geometric parameters varied and their respective levels used in the analysis of variance study.

Table 2. Results of the ANOVA analysis with respect to pressure drop.

Table 3. Results of the ANOVA analysis with respect to the mean residence time in the carbon at the rear of the carbon bed.

Table 4. Results of the ANOVA analysis with respect to the minimum residence time in the carbon at the rear of the carbon bed.

\section{APPENDIX 1: GOVERNING EQUATION AND TURBULENCE}

\section{MODELLING}

According to Reynolds decomposition each variable $q$ is split into a mean component $\bar{q}$, and a fluctuating component $q^{\prime}$.

$q=\bar{q}+q^{\prime}$

The Reynolds-averaged Navier-Stokes equations can be derived from the instantaneous Navier-Stokes equations by the Reynolds decomposition. ${ }^{(11)}$ The Reynolds-averaged mass conservation equation for incompressible flow can be expressed as:

$\frac{\partial \overline{u_{l}}}{\partial x_{i}}=0$

The Reynolds-averaged momentum conservation in the $\mathrm{i}^{\text {th }}$ direction takes the following form under statistically steady-state:

$\frac{\partial}{\partial x_{j}}\left(\rho \bar{u}_{i} \bar{u}_{j}\right)=\frac{\partial}{\partial x_{j}}\left[\mu_{l} \frac{\partial \bar{u}_{i}}{\partial x_{j}}\right]-\frac{\partial \bar{p}}{\partial x_{i}}-\frac{\partial}{\partial x_{j}}\left(\overline{\rho u_{\imath}^{\prime} u_{j}^{\prime}}\right)+\bar{S}_{i}$

Here, $\bar{S}_{i}$ is the source term in the $\mathrm{i}^{\text {th }}$ direction and in the context of current analsysis $\bar{S}_{i}$ is given by Eq. 1 . The term $\left(\overline{\rho u_{\imath}^{\prime} u_{\jmath}^{\prime}}\right)$ is the Reynold stress component, which is unclosed and needs to be modelled. Here, the Reynolds stress components are modelled here with the help of a low-Reynolds number $k-\epsilon$ turbulence model. ${ }^{(11)}$ In the framework of $k-\epsilon$ turbulence model, the Reynolds stress components $\left(\overline{\rho u_{\imath}^{\prime} u_{\jmath}^{\prime}}\right)$ are modelled as: 
$-\overline{\rho u_{\imath}^{\prime} u_{\jmath}^{\prime}}=\mu_{t}\left(\frac{\partial \bar{u}_{i}}{\partial x_{j}}+\frac{\partial \bar{u}_{j}}{\partial x_{i}}\right)-\frac{2}{3} \delta_{i j} \rho k$

where $\mu_{t}$ is the eddy viscosity, which is expressed in the following manner in the context of low Reynolds number $k-\epsilon$ turbulence model: ${ }^{(11)}$

$\mu_{t}=f_{\mu} C_{\mu} \rho k^{2} / \epsilon$

Here, $C_{\mu}=0.09$ is a model parameter and $f_{\mu}$ is a damping function which accounts for walldampening of eddy viscosity within the viscous-sublayer, which is given by: ${ }^{(11)}$

$f_{\mu}=\exp \left(-3.4 /\left(1+\frac{R e_{t}}{50}\right)^{2}\right) \quad$ where $\quad R e_{t}=\frac{\rho k^{2}}{\mu_{l} \epsilon}$

One needs to evaluate turbulent kinetic energy $k=\overline{u_{\imath}^{\prime} u_{\imath}^{\prime}} / 2$ and its dissipation rate $\epsilon=$ $\overline{v\left(\partial u_{\imath}^{\prime} / \partial x_{J}\right)\left(\partial u_{l}^{\prime} / \partial x_{J}\right)}$ in order to obtain $\mu_{t}$, and this is achieved by solving modelled transport equations of $k$ and $\epsilon$ :

$\frac{\partial}{\partial x_{j}}\left(\rho \bar{u}_{j} k\right)=\frac{\partial}{\partial x_{j}}\left[\left(\mu_{l}+\frac{\mu_{t}}{\sigma_{k}}\right) \frac{\partial k}{\partial x_{j}}\right]+\underbrace{\mu_{t}\left(\frac{\partial \bar{u}_{i}}{\partial x_{j}}+\frac{\partial \bar{u}_{i j}}{\partial x_{i}}\right) \frac{\partial \bar{u}_{i}}{\partial x_{j}}}_{\begin{array}{c}\text { Turbulent kinetic } \\ \text { energy generation }\end{array}}-\underbrace{\rho \epsilon}_{\begin{array}{c}\text { turbulent } \\ \text { dissipation }\end{array}}$

$\frac{\partial}{\partial x_{j}}\left(\rho \bar{u}_{j} \epsilon\right)=\frac{\partial}{\partial x_{j}}\left[\left(\mu_{l}+\frac{\mu_{t}}{\sigma_{\epsilon}}\right) \frac{\partial \epsilon}{\partial x_{j}}\right]+\frac{\epsilon}{k}\left[C_{\epsilon 1} \mu_{t}\left(\frac{\partial \bar{u}_{i}}{\partial x_{j}}+\frac{\partial \bar{u}_{i j}}{\partial x_{i}}\right) \frac{\partial \bar{u}_{i}}{\partial x_{j}}\right]-\frac{\rho \epsilon^{2}}{k} C_{\epsilon 2}$

Here, $\sigma_{k}, . \sigma_{\epsilon}, C_{\epsilon_{1}}$ and $C_{\epsilon 2}$ are the model coefficients, which are given by: ${ }^{(11)}$

$\sigma_{k}=1.1 \quad \sigma_{\epsilon}=1.3 \quad C_{\epsilon 1}=1.44$ and $C_{\epsilon 2}=1.92$

Turbulence at the inlet is described by two parameters, turbulence intensity $I=u^{\prime} / U$ and the integral length scale $l=C_{\mu} k^{\frac{3}{2}} / \epsilon$ where $u^{\prime}=\sqrt{2 k / 3}$ is the root mean square of velocity fluctuations, $U$ is the mean inlet velocity, and the integral length scale at the inlet was chosen as: $l=0.07 d$ where $d$ is the canister diameter. Three different turbulence intensities (i.e. $2 \%$, $10 \%$ and $20 \%$ ) have been considered for the base geometry, which showed there is a very small change in pressure drop (i.e. less than $1.0 \%$ ) even when the turbulence intensity changed by an 
order of magnitude. This is a result of the dampening of inlet turbulence close to the inlet. Thus, a turbulence intensity of $10 \%$ at the inlet has been chosen for the purpose of parametric analysis.

The turbulent kinetic energy is identically zero (i.e. $k_{\text {wall }}=0$ ) at the wall and the dissipation at the wall is specified as $\varepsilon_{\text {wall }}=2 v(\partial \sqrt{k} / \partial n)^{2}$ in the context of low Reynolds number $k-\epsilon$ turbulence model. ${ }^{(5)}$ It is worth noting that wall conditions are not used in the context of the low Reynolds number $k-\varepsilon$ model and both $k$ and $\varepsilon$ are solved all the way up to the wall. This requires adequate resolution of the viscous sub-layer of the turbulent boundary layer. It was ensured that the maximum non-dimensional wall distance $y^{+}=\rho \sqrt{\tau_{w} / \rho} \Delta / \mu_{l}$ remains smaller than 5 (i.e. $y^{+} \leq 5$ ) for all cases considered here (where $\Delta$ is the wall normal distance of the grid point next to the wall and $\sqrt{\tau_{w} / \rho}$ is the friction velocity with $\tau_{w}$ being the wall shear stress). 


\section{APPENDIX 2: MODEL CHARACTERISTICS}

Table A1. Key characteristics for each of the meshes used to establish grid independence.

\begin{tabular}{lllllll}
\hline Case & $\begin{array}{l}\text { Target } \\
\text { element } \\
\text { length } \\
\text { carbon } \\
\text { region }(\mathbf{m})\end{array}$ & $\begin{array}{l}\text { Target } \\
\text { element } \\
\text { length } \\
\text { rest } \\
\text { canister }(\mathbf{m})\end{array}$ & $\begin{array}{l}\text { Max } \\
\text { in } \\
\text { number of } \\
\text { inflation } \\
\text { layers }\end{array}$ & $\begin{array}{l}\text { Inflation layer } \\
\text { growth ratio }\end{array}$ & $\begin{array}{l}\text { Inflation layer } \\
\text { transition } \\
\text { ratio }\end{array}$ & $\begin{array}{l}\text { Number } \\
\text { of } \\
\text { in } \\
\text { cells } \\
\text { base }\end{array}$ \\
\hline Mesh A & $3 \times 10^{-4}$ & $4.5 \times 10^{-4}$ & 10 & 1.05 & & \\
Mesh B & $2.7 \times 10^{-4}$ & $4.05 \times 10^{-4}$ & 12 & 1.05 & 0.272 & 70625 \\
Mesh C & $2 \times 10^{-4}$ & $3 \times 10^{-4}$ & 15 & 1.05 & 0.272 & 85323 \\
\hline
\end{tabular}

Table A2. Permeabilities of the materials used for the 3D simulations

\begin{tabular}{ll}
\hline Material & Permeability $\left(\mathbf{m}^{-\mathbf{2}}\right)$ \\
\hline Filter paper & $1.52 \times 10^{9}$ \\
Carbon & $2.39 \times 10^{9}$ \\
\hline
\end{tabular}

Table A3. Experimental and computational pressure drops across the 3D simulation for a flow rate of $50 \mathrm{~L} / \mathrm{min}$.

\begin{tabular}{ll}
\hline Case & Pressure drop (Pa) \\
\hline Experimental & 250 \\
Fixed carbon permeability & 225 \\
Carbon porosity described by Mueller & 241 \\
equation & \\
\hline
\end{tabular}

Table A4. Hardware used for CFD simulations.

\begin{tabular}{ll}
\hline CPU & Intel i7-6700K, $(8$ CPU cores at $~ 4 \mathrm{GHz})$ \\
GPU & NVIDIA Quadro K620 4GB \\
RAM & 32 GB DDR3 \\
\hline
\end{tabular}




\section{APPENDIX 3: FILTER EQUATIONS}

A pressure drop occurs as fluid flows through a porous medium due to both viscous and inertial effects as described by the Forchheimer equation: $(-\Delta \bar{p} / L)=a_{1} V_{s}+a_{2} V_{s}^{2}$ where $a_{1}$ and $a_{2}$ are constants which depend upon the porous medium and $V_{s}$ is the superficial fluid velocity entering into the porous filter material. The term $a_{1} V_{s}$ represents viscous effects, whereas $a_{2} V_{s}^{2}$ accounts for inertial effects. It has previously been shown that the inertial term only becomes significant at higher values of $R e^{\prime}=a_{2} \rho \bar{u}_{i} / a_{1} \mu \cdot{ }^{(8)}$ For the flowrates studied here $R e^{\prime}$ was of the order of $10^{-2}$, where the inertial term has been shown to be negligible and thus the Forchheimer equation reduces to Darcy's law. ${ }^{(8)}$ The mean pressure gradient in the $\mathrm{i}^{\text {th }}$ direction in a porous medium of permeability $K$ can therefore be given by Darcy's law: ${ }^{(19)}$

$\left(\frac{-\Delta \bar{p}}{L}\right)_{i}=\frac{\mu}{K} \bar{u}_{i}$

In the carbon bed the local permeability can be found from the Ergun equation. ${ }^{(12)}$ The Ergun equation is valid for a uniformly packed bed of known porosity $E_{p}$ and a single particle diameter $d_{p}$ :

$K=d_{p}^{2} E_{p}^{2} / 150 E_{p}^{3}$

The porosity throughout the carbon bed varies in the radial direction as a result of the imperfect packing of the carbon granules close to the canister walls, as given by the Mueller equation: ${ }^{(14)}$

$E_{p}=E_{b}\left(1-E_{b}\right) e^{-b r^{*}} J_{0}\left(a r^{*}\right)$

where $d_{p}$ is the bead diameter, $d_{b}$ is the bed diameter, $r$ is the radial position in the bed, $E_{p}$ is the voidage and $J_{0}$ is a zero order Bessel function of the first kind. The parameters $a, b$ and $r^{*}$ are given by:

$a=8.0-\frac{3.15 d_{p}}{d_{b}}$ for $2.02 \leq \frac{d_{b}}{d_{p}} \leq 13.0$ 


$$
\begin{aligned}
& a=8.0-\frac{11.25 d_{p}}{d_{b}} \text { for } 13 \leq \frac{d_{b}}{d_{p}} \\
& b=0.315-\frac{0.725 d_{b}}{d_{p}} \\
& E_{b}=0.334+\frac{0.220 d_{p}}{d_{b}} \\
& r^{*}=r / d_{p}
\end{aligned}
$$

